\title{
Robust response-guided dosing
}

\author{
Saumya Sinha \\ Jakob Kotas \\ Archis Ghate*
}

March 22, 2016

\begin{abstract}
In response-guided dosing (RGD), doses are adapted to the uncertain progression of each patient's disease condition. A stochastic dynamic program was recently developed for RGD. We study its robust counterpart, where the dose-response distribution belongs to an uncertainty set. For interval uncertainty sets, we prove that it is optimal to administer higher doses in worsening disease. When a certain scaling of a nominal distribution describes the interval, optimal doses also increase in the scaling parameter. Theory is illustrated via numerical results.
\end{abstract}

Keywords: stochastic dose-response; robust Markov decision processes; medical treatment planning

\section{Background and motivation}

Many diseases are treated with therapeutic drugs over multiple sessions where dosing guidelines follow a one-size-fits-all philosophy. For instance, a daily radiation dose of 2 Gy for 35 days is recommended for head-and-neck cancer [7], and a weekly dose of $180 \mu \mathrm{g}$ interferon for 48 weeks is prescribed for hepatitis C [9]. While such guidelines are easy to implement, their potential drawbacks include inadequate disease control, over- and under-dosing, and unsatisfactory cost-effectiveness [4]. Response-guided dosing (RGD) is an alternative paradigm, where doses are adapted to the observed uncertain evolution of a patient's disease condition. RGD attempts to balance a fundamental trade-off in medicine - higher doses achieve better disease-control at the cost of undesirable side-effects, whereas lower doses sacrifice disease-control in favor of lesser adverse effects.

Most clinical implementations of RGD are, however, somewhat ad hoc. Indeed, Murphy et al. [8] have commented: "despite the activity in evaluating adaptive treatment strategies, the development of data collection and analytic methods that directly inform the construction of adaptive treatment strategies lags behind." To address this concern, a stochastic dynamic programming (DP) framework for choosing doses in RGD was recently proposed by our research group in Kotas and Ghate [6]. The idea there was to balance the total disutility of doses delivered over the treatment course against the disease condition reached by the end of the treatment course. The evolution of this disease condition was characterized using a stochastic dose-response model - a formula that yields the next disease condition as a function of the current disease condition and of the dose used in the current treatment session, subject to uncertainty in a dose-response parameter. Kotas and

${ }^{*}$ Corresponding author: Industrial and Systems Engineering, Box 352650, University of Washington, Seattle, WA 98195, USA; archis@uw.edu 
Ghate proved, under natural convexity and monotonicity assumptions on this formula and on the disutility functions, that optimal doses are increasing in worsening disease conditions.

One limitation of this stochastic DP is that the decision-maker is assumed to know at the outset the probability mass function (pmf) of the dose-response parameter. Any a priori estimate of this pmf, however, is subject to estimation errors. To tackle the resulting ambiguity, we present here a robust counterpart of the Kotas and Ghate model (henceforth called the "nominal" model).

In our robust formulation, the pmf of the dose-response parameter will be assumed to belong to an uncertainty set. Uncertainty sets are often composed of pmfs that are in some sense "close to" a nominal pmf, which may have been estimated a priori from a clinical trial $[1,5,10]$. Roughly speaking, the decision-maker then follows a conservative approach whereby he/she attempts to find a dosing policy that minimizes the worst-case expected disutility over all pmfs from this uncertainty set. Examples of uncertainty sets include the interval set, the maximum likelihood set, the relative entropy set, and the ellipsoidal set. Although our general robust RGD model accommodates any of these sets, we illustrate our results in detail using the interval model. We show that the socalled inner maximization problem in the Bellman's equations for robust RGD with the interval uncertainty set is a linear program (LP) that can be solved analytically. Moreover, an optimal solution to this inner problem, that is, the worst-case pmf, does not depend on the observed disease condition and the dose chosen. This in turn implies that there exists a monotone dosing policy that is optimal to the robust stochastic DP, thus extending the main theoretical result from the nominal model of Kotas and Ghate (see Section 3 below). This extension is not only of theoretical interest but also significantly simplifies the computation of our robust optimal dosing policy. In particular, we show that the state-action invariant structure of the worst-case pmf makes the robust problem only as hard to solve as the nominal problem. We further analyze in Section 4 a specific and common single-parameter formulation of the interval uncertainty set and provide a simple condition on the dose-response formula under which optimal doses vary monotonically with this parameter. We conclude by presenting numerical results on a hypothetical disease with an inverse-power dose-response function.

\section{Review of the nominal model}

Let $T$ denote the number of sessions, indexed by $t=1,2, \ldots, T$, in a treatment course. At the beginning of each session, the decision-maker observes a numerical score of the patient's disease condition, and chooses a dose for that session. These numerical scores belong to a compact interval $X \subseteq \mathbb{R}$. Smaller numbers in this set represent less severe disease. The disease condition at the beginning of session $t$ is denoted by $x_{t} \in X$. The dose level chosen by the decision-maker for this session after observing $x_{t}$ is denoted by $d_{t}$. Dose levels $d_{t}$ belong to the interval $D \triangleq[0, \bar{d}] \subset \mathbb{R}$, where $\bar{d}$ is a finite upper bound on permissible dose levels.

For $t=1,2, \ldots, T$, disease conditions evolve according to dynamics $x_{t+1}=x_{t}+f\left(d_{t} ; \Theta_{t}\right)$, for $x_{t}, x_{t+1} \in X$ and $d_{t} \in D$. All standard dose-response functions such as linear, Michaelis-Menten, inverse-power, Emax, Hill's, exponential, exponential linear-quadratic, power law, Gompertz, and Beta-Poisson can be expressed in this form (after taking logarithms in some cases). Please see Kotas and Ghate and references therein for detailed descriptions of these functions. Here, $\Theta_{t}$ are independent and identically distributed dose-response parameters in sessions $t$. Independence across sessions is somewhat restrictive although common in the literature (see, for example, Chapter 4 of [3], and also [11]). Kotas and Ghate employed this assumption in their nominal model as well, 
and it holds when consecutive sessions are "sufficiently separated" from a biochemical viewpoint. Random variables $\Theta_{t}$ take values from a finite set $\Omega \triangleq\left\{\theta_{1}, \theta_{2}, \ldots, \theta_{n}\right\}$. Their pmf, denoted by $p(\cdot)$, is known to the decision-maker. We assume that the function $f(\cdot ; \theta)$ is continuous over $D$ for each $\theta \in \Omega$.

Aversion to dose is modeled using a continuous disutility function $c: D \rightarrow \mathbb{R}_{+}$. Since $D$ is compact, continuity of $c(\cdot)$ implies that it is bounded. Examples include linear, quadratic, and exponential functions. Aversion to disease conditions $x_{T+1}$ at the end of the treatment course is modeled using a continuous and bounded disutility function $h: X \rightarrow \mathbb{R}_{+}$. Examples include linear, quadratic, exponential, and ramp (where the disutility is zero up to a disease-condition threshold and grows linearly thereafter).

Let $J_{t}\left(x_{t}\right)$ denote the minimum total expected disutility accumulated by the end of the treatment course, given that the disease condition at the beginning of the th session is $x_{t}$. These optimal cost-to-go functions $J_{t}(\cdot)$ are unique solutions of Bellman's equations

$$
J_{t}\left(x_{t}\right)=\min _{d_{t} \in D}\left\{c\left(d_{t}\right)+\sum_{\theta \in \Omega} J_{t+1}\left(x_{t}+f\left(d_{t} ; \theta\right)\right) p(\theta)\right\}, \forall x_{t} \in X, \text { and } t=1,2, \ldots, T,
$$

with the boundary condition $J_{T+1}(x)=h(x)$ for all $x \in X$. Problem (1) involves optimizing a continuous function over the nonempty compact set $D$ and hence it has an optimal solution. Doses that attain the above minima define an optimal RGD policy. Bellman's equations (1) can easily be solved approximately using discretization of $X$ and $D$. As such, the nominal problem is computationally tractable. Moreover, it is shown in Kotas and Ghate, under two assumptions (stated below) on the dose-response function and on the disutility functions, that in each treatment session there exist optimal doses that increase as the disease condition worsens.

Assumption 2.1 (Monotone and convex dose-response). The function $f(\cdot ; \theta)$ is decreasing and convex in dose over $D$ for every $\theta \in \Omega$.

Assumption 2.2 (Increasing and convex disutilities). The disutility function $c(\cdot)$ is increasing and convex over $D$; the disutility function $h(\cdot)$ is increasing and convex over $X$.

A detailed justification for these assumptions was provided by Kotas and Ghate. In particular, Assumption 2.2 encodes a risk-averse decision-maker. Several examples of clinically relevant functions that satisfy these assumptions were also listed by Kotas and Ghate; as such, we do not believe these assumptions to be particularly restrictive.

Theorem 2.3 (Theorem 5.3 in Kotas and Ghate). Under the above assumptions, optimal dose levels increase with worsening disease conditions in each treatment session.

\section{The robust stochastic DP}

As opposed to the nominal problem, we now consider the case where the pmf of the dose-response parameter is not known to the decision-maker. Pursuing standard practice in robust stochastic DP $[1,5,10]$, we assume that the pmf of $\Theta_{t}$ is only known to lie in some set $\mathcal{P}$. In the robust optimization parlance, set $\mathcal{P}$ is called the uncertainty set and is composed of all "plausible" pmfs. This set is often chosen so that it includes all pmfs that are "close to" some nominal pmf. More precisely, let 
$\Delta \triangleq\left\{p(\cdot): p(\theta) \geq 0, \sum_{\theta \in \Omega} p(\theta)=1\right\}$ be the probability simplex in $\mathbb{R}^{n}$, and let $\mathcal{P} \subseteq \Delta$. Then, the worst-case total expected disutility is minimized by solving the robust Bellman's equations

$$
\tilde{J}_{t}\left(x_{t}\right)=\min _{d_{t} \in D}\{\overbrace{\max _{p_{t}(\cdot) \in \mathcal{P}}\left(c\left(d_{t}\right)+\sum_{\theta \in \Omega} \tilde{J}_{t+1}\left(x_{t}+f\left(d_{t} ; \theta\right)\right) p_{t}(\theta)\right)}^{\text {"inner problem" }}\}, \forall x_{t} \in X \text {, and } t=1,2, \ldots, T,
$$

with the boundary condition $\tilde{J}_{T+1}(x)=h(x)$ for all $x \in X$. Here, $\tilde{J}_{t}(\cdot)$, for $t=1,2, \ldots, T+1$, are called the robust optimal cost-to-go functions, and an optimal solution to the inner problem is called a worst-case distribution.

The robust stochastic DP is computationally tractable if the inner maximization problem is easy to solve. This occurs, for example, when $\mathcal{P}$ is chosen to be a convex set. This, combined with the linearity (in $p_{t}(\cdot)$ ) of the objective function, implies that the inner problem is convex. Some examples of convex uncertainty sets are interval, maximum likelihood, ellipsoidal and entropy $[1,5,10]$. We focus on the interval uncertainty model in the subsequent discussion.

The interval model is motivated by statistical estimates of confidence intervals on the pmf components. It can also be obtained by projecting the ellipsoidal or maximum likelihood uncertainty sets onto the coordinate axes $[1,10]$. In this model [1], the uncertainty set $\mathcal{P}$ is defined such that the probabilities $p(\theta)$, for $\theta \in \Omega$, belong to an interval. More precisely, $\mathcal{P} \triangleq\left\{p(\theta) \in \Delta: p_{L}(\theta) \leq\right.$ $\left.p(\theta) \leq p_{H}(\theta)\right\}$, for some constants $p_{L}(\theta)$ and $p_{H}(\theta)$, for $\theta \in \Omega$. We assume, to avoid trivialities, that $\mathcal{P}$ is non-empty. A necessary condition for this is that $\sum_{\theta \in \Omega} p_{L}(\theta) \leq 1$ and $\sum_{\theta \in \Omega} p_{H}(\theta) \geq 1$.

In the interval uncertainty model, the inner problem is an LP, and in fact, we are able to find a closed-form solution for this LP. Moreover, it turns out that the resulting worst-case pmf does not depend on $t, x_{t}$, or $d_{t}$. This implies that the robust problem is as easy to solve as the nominal problem and it also enables us to prove a monotonicity result in Theorem 3.2 below, analogous to Theorem 2.3 from the nominal case, under the following additional assumption.

Assumption 3.1 (Monotonicity in dose-response parameter). The dose-response function $f(d ; \cdot)$ is decreasing over $\Omega$ for each $d \in D$.

This assumption implies that larger dose-response parameters induce a larger improvement in the disease condition. Our proofs below can be rewritten for a variation of this assumption where the improvement is increasing in the dose-response parameter. All dose-response models known to us satisfy one of these two types of monotonicity; Assumption 3.1 thus is not restrictive.

Theorem 3.2. Suppose Assumptions 2.1, 2.2, and 3.1 hold. Then, optimal dose levels increase with worsening disease conditions in each session in the robust stochastic DP with interval uncertainty.

Proof of Theorem 3.2: The proof employs backward induction on $t=T+1, T, \ldots, 1$. We first show that if $J_{t+1}(\cdot)$ is increasing, convex, continuous, and bounded, then in treatment session $t$, there exist optimal doses that are monotone. We then show that $J_{t}(\cdot)$ inherits these four properties from $J_{t+1}(\cdot)$. The theorem then follows because the utility function $h(\cdot)$, that is, $J_{T+1}(\cdot)$, is assumed to possess these properties.

So suppose, as the inductive hypothesis, that $\tilde{J}_{t+1}(\cdot)$ is increasing, convex, continuous, and bounded. For each fixed state-action pair $\left(x_{t}, d_{t}\right)$, the inner problem

$$
\tilde{Q}_{t}\left(x_{t}, d_{t}\right) \triangleq \max _{p_{t}(\cdot) \in \mathcal{P}}\left(c\left(d_{t}\right)+\sum_{\theta \in \Omega} \tilde{J}_{t+1}\left(x_{t}+f\left(d_{t} ; \theta\right)\right) p_{t}(\theta)\right)
$$


is an $n$-variable LP in variables $p_{t}(\theta)$, for $\theta \in \Omega$. This LP has an optimal solution because its feasible region $\mathcal{P}$ is compact. We denote this optimal solution by $p_{t}^{*}\left(x_{t}, d_{t} ; \theta\right)$, for $\theta \in \Omega$. With this notation, we have that the robust optimal cost-to-go functions are given by

$$
\tilde{J}_{t}\left(x_{t}\right)=\min _{d_{t} \in D} \tilde{Q}_{t}\left(x_{t}, d_{t}\right)=\min _{d_{t} \in D}\left(c\left(d_{t}\right)+\sum_{\theta \in \Omega} \tilde{J}_{t+1}\left(x_{t}+f\left(d_{t} ; \theta\right)\right) p_{t}^{*}\left(x_{t}, d_{t} ; \theta\right)\right) .
$$

Claim 1: Problem (3) has an optimal solution that does not depend on $t,\left(x_{t}, d_{t}\right)$ and hence $p_{t}^{*}\left(x_{t}, d_{t} ; \theta\right)$ can in fact be written as $p^{*}(\theta)$ for $\theta \in \Omega$.

Proof of Claim 1: For any fixed session $t$ and fixed state-action pair $\left(x_{t}, d_{t}\right)$, the inner maximization problem is equivalent to the LP

$$
\begin{aligned}
\max g(p) & \triangleq \sum_{\theta \in \Omega} \tilde{J}_{t+1}\left(x_{t}+f\left(d_{t} ; \theta\right)\right) p(\theta) \\
p_{L}(\theta) & \leq p(\theta) \leq p_{H}(\theta) \text { and } 0 \leq p(\theta) \leq 1, \text { for } \theta \in \Omega ; \sum_{\theta \in \Omega} p(\theta)=1 .
\end{aligned}
$$

We henceforth simplify constraints (6) by assuming without loss of generality that $0 \leq p_{L}(\theta) \leq$ $p_{H}(\theta) \leq 1$ for all $\theta \in \Omega$. Suppose, without loss of generality, that the elements of $\Omega$ are organized in ascending order as $\theta_{1} \leq \ldots \leq \theta_{n}$. Then, Assumption 3.1, combined with the fact that $\tilde{J}_{t+1}(\cdot)$ is increasing, implies that the coefficients $a\left(\theta_{i}\right) \triangleq \tilde{J}_{t+1}\left(x_{t}+f\left(d_{t}, \theta_{i}\right)\right)$ of the variables $p\left(\theta_{i}\right)$ in the LP objective function $g(\cdot)$, are decreasing in $i=1,2, \ldots, n$. Since $a\left(\theta_{1}\right)$ is the largest coefficient, we would like to attach the largest possible probability mass to $\theta_{1}$. Thus, if there is a feasible pmf $p(\cdot)$ for which $p\left(\theta_{1}\right)=p_{H}\left(\theta_{1}\right)$, we choose $p^{*}\left(\theta_{1}\right)=p_{H}\left(\theta_{1}\right)$. Otherwise, we still choose the largest feasible value that $p\left(\theta_{1}\right)$ may take and continue. This idea helps us claim that the solution $p^{*}(\cdot)$ defined below in Equation (8) is optimal. But first, we need some additional notation. Let $k$ be the smallest index (called the "switching index") in $\{1,2, \ldots, n\}$ such that

$$
\sum_{i=1}^{k} p_{H}\left(\theta_{i}\right)+\sum_{i=k+1}^{n} p_{L}\left(\theta_{i}\right) \geq 1
$$

Also let $r_{k}=1-\sum_{i=1}^{k-1} p_{H}\left(\theta_{i}\right)-\sum_{i=k+1}^{n} p_{L}\left(\theta_{i}\right)$. We will prove below that the pmf

$$
p^{*}\left(\theta_{i}\right)= \begin{cases}p_{H}\left(\theta_{i}\right), & \text { if } i \leq k-1 \\ r_{k}, & \text { if } i=k \\ p_{L}\left(\theta_{i}\right), & \text { if } i \geq k+1\end{cases}
$$

schematically illustrated in Figure 1, is optimal for the above LP.
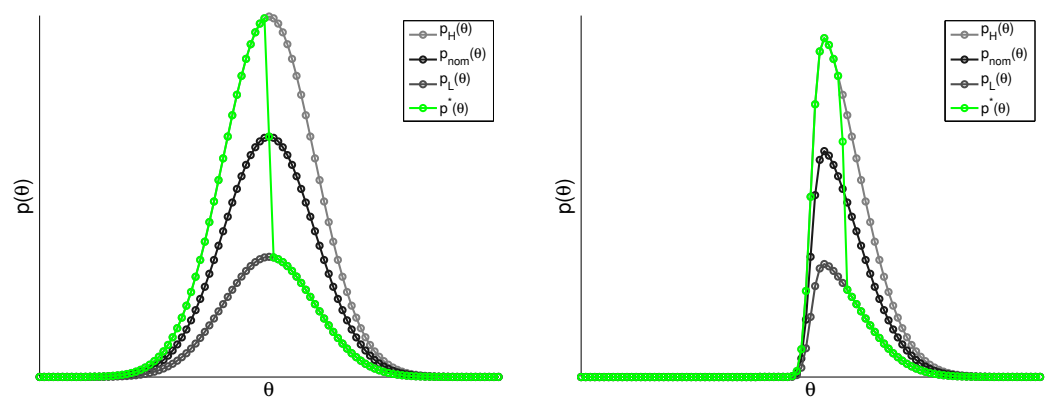

Figure 1: Illustration of the worst-case pmf defined in Equation (8) for two different nominal pmfs. 
To show that (8) is optimal to (5), observe first that $p^{*}(\cdot)$ is well-defined since $k=n$ satisfies condition (7). Also, by construction, the components $p^{*}\left(\theta_{i}\right)$ sum up to 1 . Moreover, components $p^{*}\left(\theta_{i}\right)$ for $i=1,2, \ldots, k-1$ and $i=k+1, k+2, \ldots, n$ are, by construction, between $p_{L}\left(\theta_{i}\right)$ and $p_{H}\left(\theta_{i}\right)$. So, for $p^{*}(\cdot)$ to be feasible, we need to check whether $p_{L}\left(\theta_{k}\right) \leq r_{k} \leq p_{H}\left(\theta_{k}\right)$. We do this as follows. Recall that $k$ is the smallest index for which (7) holds. Therefore, by the definition of $r_{k}$, we have, $r_{k}=1-\sum_{i=1}^{k-1} p_{H}\left(\theta_{i}\right)-\sum_{i=k+1}^{n} p_{L}\left(\theta_{i}\right) \leq p_{H}\left(\theta_{k}\right)$. Now, to show that $r_{k} \geq p_{L}\left(\theta_{k}\right)$, we consider two cases. Firstly, if $k=1$, then $r_{1}=1-\sum_{i=2}^{n} p_{L}\left(\theta_{i}\right) \geq p_{L}\left(\theta_{1}\right)$ by the necessary condition for $\mathcal{P}$ to be non-empty. Secondly, if $k \neq 1$, we know that $k-1$ does not satisfy (7). Therefore,

$$
\sum_{i=1}^{k-1} p_{H}\left(\theta_{i}\right)+\sum_{i=k}^{n} p_{L}\left(\theta_{i}\right)<1 \Rightarrow p_{L}\left(\theta_{k}\right)<1-\sum_{i=1}^{k-1} p_{H}\left(\theta_{i}\right)-\sum_{i=k+1}^{n} p_{L}\left(\theta_{i}\right)=r_{k} .
$$

Thus, we have shown that $p_{L}\left(\theta_{k}\right) \leq r_{k} \leq p_{H}\left(\theta_{k}\right)$ as required for feasibility of $p^{*}(\cdot)$.

Now, to prove optimality, let $p(\cdot)$ be any feasible solution of $(5)$. Then,

$$
\begin{aligned}
g\left(p^{*}\right)-g(p)= & \sum_{i=1}^{n} a\left(\theta_{i}\right)\left(p^{*}\left(\theta_{i}\right)-p\left(\theta_{i}\right)\right) \\
= & \sum_{i=1}^{k-1} a\left(\theta_{i}\right)\left(p_{H}\left(\theta_{i}\right)-p\left(\theta_{i}\right)\right)+a\left(\theta_{k}\right)\left(1-\sum_{i=1}^{k-1} p_{H}\left(\theta_{i}\right)-\sum_{i=k+1}^{n} p_{L}\left(\theta_{i}\right)-p\left(\theta_{k}\right)\right) \\
& +\sum_{i=k+1}^{n} a\left(\theta_{i}\right)\left(p_{L}\left(\theta_{i}\right)-p\left(\theta_{i}\right)\right) \\
= & \sum_{i=1}^{k-1} a\left(\theta_{i}\right)\left(p_{H}\left(\theta_{i}\right)-p\left(\theta_{i}\right)\right)+\sum_{i=k+1}^{n} a\left(\theta_{i}\right)\left(p_{L}\left(\theta_{i}\right)-p\left(\theta_{i}\right)\right) \\
& +a\left(\theta_{k}\right)\left(-\sum_{i=1}^{k-1} p_{H}\left(\theta_{i}\right)-\sum_{i=k+1}^{n} p_{L}\left(\theta_{i}\right)+\sum_{i=1}^{k-1} p\left(\theta_{i}\right)+\sum_{i=k+1}^{n} p\left(\theta_{i}\right)\right) \\
& k-1 \\
= & \sum_{i=1}^{k-1}\left(a\left(\theta_{i}\right)-a\left(\theta_{k}\right)\right)\left(p_{H}\left(\theta_{i}\right)-p\left(\theta_{i}\right)\right)+\sum_{i=k+1}^{n}\left(a\left(\theta_{i}\right)-a\left(\theta_{k}\right)\right)\left(p_{L}\left(\theta_{i}\right)-p\left(\theta_{i}\right)\right) \geq 0 .
\end{aligned}
$$

Here, the last inequality holds because each summand is nonnegative. Hence, $p^{*}(\cdot)$ as described in (8) is optimal. Furthermore, we emphasize that this optimal solution does not depend on $t, x_{t}$, or $d_{t}$. This is because the above proof of optimality only uses the fact that $a\left(\theta_{j}\right)-a\left(\theta_{i}\right) \geq 0$ for $j>i$, and in particular, does not utilize the specific values of these coefficients. In other words, this optimal solution depends only on the ordering of the coefficients $a\left(\theta_{i}\right)$, which is invariant with respect to $t, x_{t}, d_{t}$. We thus rewrite $(3)$ as $\tilde{Q}_{t}\left(x_{t}, d_{t}\right)=c\left(d_{t}\right)+\sum_{\theta \in \Omega} \tilde{J}_{t+1}\left(x_{t}+f\left(d_{t} ; \theta\right)\right) p^{*}(\theta)$.

\section{End of Proof of Claim 1.}

Proofs of the next three claims are identical to similar results in Kotas and Ghate.

Claim 2: $\tilde{Q}_{t}(\cdot ; \cdot)$ has decreasing differences over $X \times D$, and hence optimal doses in session $t$ are increasing in disease conditions. 
Proof of Claim 2: Consider any $x^{+} \geq x^{-}$and $d^{+} \geq d^{-}$. Then $\tilde{Q}_{t}\left(x^{+} ; d^{+}\right)-\tilde{Q}_{t}\left(x^{+} ; d^{-}\right)=$ $c\left(d^{+}\right)-c\left(d^{-}\right)+\sum_{\theta \in \Omega}\left(\tilde{J}_{t+1}\left(x^{+}+f\left(d^{+} ; \theta\right)\right)-\tilde{J}_{t+1}\left(x^{+}+f\left(d^{-} ; \theta\right)\right)\right) p^{*}(\theta) \leq c\left(d^{+}\right)-c\left(d^{-}\right)+\sum_{\theta \in \Omega}\left(\tilde{J}_{t+1}\left(x^{-}+\right.\right.$ $\left.\left.f\left(d^{+} ; \theta\right)\right)-\tilde{J}_{t+1}\left(x^{-}+f\left(d^{-} ; \theta\right)\right)\right) p^{*}(\theta)=\tilde{Q}_{t}\left(x^{-} ; d^{+}\right)-\tilde{Q}_{t}\left(x^{-} ; d^{-}\right)$. Here, the inequality follows because $f\left(d^{+} ; \theta\right) \leq f\left(d^{-} ; \theta\right)$ by the first half of Assumption 2.1 and because $\tilde{J}_{t+1}(\cdot)$ is increasing and convex. This proves decreasing differences, which imply monotonicity of optimal doses via a standard argument by contradiction.

\section{End of Proof of Claim 2.}

Claim 3: $\tilde{J}_{t}(\cdot)$ is increasing in disease condition.

Proof of Claim 3: Suppose dose $d^{+}$is optimal in state $x^{+}$and dose $d^{-}$is optimal in state $x^{-} \leq x^{+}$. Then, $\tilde{J}_{t}\left(x^{+}\right)=\tilde{Q}_{t}\left(x^{+} ; d^{+}\right)=c\left(d^{+}\right)+\sum_{\theta \in \Omega}\left(\tilde{J}_{t+1}\left(x^{+}+f\left(d^{+} ; \theta\right)\right) p^{*}(\theta) \geq c\left(d^{+}\right)+\sum_{\theta \in \Omega}\left(\tilde{J}_{t+1}\left(x^{-}+\right.\right.\right.$ $\left.f\left(d^{+} ; \theta\right)\right) p^{*}(\theta)=\tilde{Q}_{t}\left(x^{-} ; d^{+}\right) \geq \tilde{Q}_{t}\left(x^{-} ; d^{-}\right)=\tilde{J}_{t}\left(x^{-}\right)$. Here, the first inequality holds because $\tilde{J}_{t+1}(\cdot)$ is increasing and the second inequality holds because $d^{-}$is optimal in $x^{-}$.

\section{End of Proof of Claim 3.}

Claim 4: $\tilde{Q}_{t}(\cdot ; \cdot)$ is convex over $X \times D$, and $\tilde{J}_{t}(\cdot)$ is convex over $X$.

Proof of Claim 4: First note that $\tilde{J}_{t+1}\left(x_{t}+f\left(d_{t} ; \theta\right)\right)$ is convex over $X \times D$ for every $\theta \in \Omega$. This holds because $\tilde{J}_{t+1}(\cdot)$ is an increasing convex function and $x_{t}+f\left(d_{t} ; \theta\right)$ is convex by the second half of Assumption 2.1 (see Section 3.2.4 of [2]). Thus, $\sum_{\theta \in \Omega} \tilde{J}_{t+1}\left(x_{t}+f\left(d_{t} ; \theta\right)\right) p_{t}^{*}(\theta)$ is also convex because expectation preserves convexity. Convexity of $\tilde{Q}_{t}(\cdot ; \cdot)$ then follows because $c(\cdot)$ is convex by Assumption 2.2. Finally, $\tilde{J}_{t}(\cdot)$ is convex because convexity is preserved under minimization (see Section 3.2 .5 of $[2])$.

\section{End of Proof of Claim 4.}

Claim 5: $\tilde{Q}_{t}(\cdot ; \cdot)$ and $\tilde{J}_{t}(\cdot)$ are continuous and bounded.

Proof of Claim 5: By a technical argument identical to Kotas and Ghate; omitted. End of Proof of Claim 5.

Claims 2, 3, 4, and 5 restore the inductive hypothesis.

\section{End of Proof of Theorem 3.2.}

In addition to facilitating the above proof of monotonicity, the worst-case pmf in (8) offers a computational advantage. Since this worst-case pmf does not depend on $t, x_{t}$, or $d_{t}$, we only need to find it once at the outset of the Bellman's recursion (2). This reduces the robust Bellman's equations to those in a nominal stochastic DP where the pmf equals the worst-case pmf (8).

\section{Monotonicity with respect to ambiguity level}

We now further analyze one common interval uncertainty set that is characterized by a single parameter $0 \leq \delta \leq 1$ (see [1]). Specifically, the lower and upper bounds are chosen as

$$
p_{L}(\theta)=(1-\delta) p_{\text {nom }}(\theta), \quad \text { and } \quad p_{H}(\theta)=(1+\delta) p_{\text {nom }}(\theta), \quad \text { for all } \theta \in \Omega
$$


where $p_{\text {nom }}(\cdot)$ is some nominal pmf over $\Omega$. In Theorem 4.2 below, we prove that optimal doses are monotonically increasing in $\delta$ under the following assumption.

Assumption 4.1. The dose-response function $f(\cdot ; \cdot)$ has increasing differences over $D \times \Omega$; that is, for $d^{+} \geq d^{-}$and for $\theta^{+} \geq \theta^{-}, f\left(d^{+} ; \theta^{+}\right)-f\left(d^{-} ; \theta^{+}\right) \geq f\left(d^{+} ; \theta^{-}\right)-f\left(d^{-} ; \theta^{-}\right)$.

Theorem 4.2. Suppose the dose response function satisfies all assumptions of Theorem 3.2, as well as Assumption 4.1. Then robust optimal doses are increasing in $\delta$.

Let us first examine the worst-case $\operatorname{pmf} p_{t}^{*}(\cdot)$ for this interval uncertainty model. Recall that the switching index $k$ is the smallest integer in $\{1,2, \ldots, n\}$ which satisfies

$$
\sum_{i=1}^{k}(1+\delta) p_{\mathrm{nom}}\left(\theta_{i}\right)+\sum_{i=k+1}^{n}(1-\delta) p_{\mathrm{nom}}\left(\theta_{i}\right) \geq 1 .
$$

For $\delta=0$, this is true for any integer $m$ and the worst-case distribution is the same as the nominal distribution. For $0<\delta \leq 1$, we show that the switching index $k$ is independent of $\delta$ and depends only on the nominal pmf. This holds because of the following algebraic argument. Consider any $0<\delta \leq 1$ and any index $m$ between 1 and $n$. Then,

$$
\begin{gathered}
(1-\delta) \sum_{i=1}^{m} p_{\text {nom }}\left(\theta_{i}\right)+(1+\delta) \sum_{i=m+1}^{n} p_{\text {nom }}\left(\theta_{i}\right) \geq 1 \\
\text { iff } \delta\left[-\sum_{i=1}^{m} p_{\text {nom }}\left(\theta_{i}\right)+\sum_{i=m+1}^{n} p_{\text {nom }}\left(\theta_{i}\right)\right] \geq 0 \\
\quad \text { iff } \sum_{i=m+1}^{n} p_{\text {nom }}\left(\theta_{i}\right) \geq \sum_{i=1}^{m} p_{\text {nom }}\left(\theta_{i}\right) .
\end{gathered}
$$

Thus, condition (9) is equivalent to inequality (10), which depends only on the nominal pmf. Thus, the switching index $k$ does not depend on the parameter $\delta$.

Now, to examine the dependence of the optimal dose on $\delta$, we define the function $W_{t}(d, \delta) \triangleq$ $c(d)+\sum_{\theta \in \Omega} J_{t+1}(x+f(d ; \theta)) p^{*}(\theta)$; this equals the worst-case expected value for a fixed dose $d$ when the uncertainty is parametrized by $\delta$. For this discussion, the state $x_{t}$ is assumed to be fixed and hence we have suppressed the dependence of $W_{t}$ on $x_{t}$ in our notation. The robust optimal cost-togo function is obtained by minimizing $W_{t}(\cdot, \delta)$ over all doses $d$. First, we derive an expression for $W_{t}$ that is convenient for the rest of our proof below (we suppress the subscript nom for brevity).

$$
\begin{aligned}
& W_{t}(d, \delta)=c(d)+(1+\delta) \sum_{i=1}^{k-1} \tilde{J}_{t+1}\left(x+f\left(d ; \theta_{i}\right)\right) p\left(\theta_{i}\right)+(1-\delta) \sum_{i=k+1}^{n} \tilde{J}_{t+1}\left(x+f\left(d ; \theta_{i}\right)\right) p\left(\theta_{i}\right) \\
& +\left(1-\sum_{i=1}^{k-1}(1+\delta) p\left(\theta_{i}\right)-\sum_{i=k+1}^{n}(1-\delta) p\left(\theta_{i}\right)\right) \tilde{J}_{t+1}\left(x+f\left(d ; \theta_{k}\right)\right) \\
& =c(d)+(1+\delta) \sum_{i=1}^{k-1} \tilde{J}_{t+1}\left(x+f\left(d ; \theta_{i}\right)\right) p\left(\theta_{i}\right)+(1-\delta) \sum_{i=k+1}^{n} \tilde{J}_{t+1}\left(x+f\left(d ; \theta_{i}\right)\right) p\left(\theta_{i}\right) \\
& +\left(p\left(\theta_{k}\right)-\delta\left(\sum_{i=1}^{k-1} p\left(\theta_{i}\right)-\sum_{i=k+1}^{n} p\left(\theta_{i}\right)\right)\right) \tilde{J}_{t+1}\left(x+f\left(d ; \theta_{k}\right)\right) \\
& =c(d)+\sum_{i=1}^{n} \tilde{J}_{t+1}\left(x+f\left(d ; \theta_{i}\right)\right) p\left(\theta_{i}\right)+\delta \sum_{i=1}^{k-1} \tilde{J}_{t+1}\left(x+f\left(d ; \theta_{i}\right)\right) p\left(\theta_{i}\right)
\end{aligned}
$$




$$
\begin{aligned}
& -\delta \sum_{i=k+1}^{n} \tilde{J}_{t+1}\left(x+f\left(d ; \theta_{i}\right)\right) p\left(\theta_{i}\right)-\delta\left(\sum_{i=1}^{k-1} p\left(\theta_{i}\right)-\sum_{i=k+1}^{n} p\left(\theta_{i}\right)\right) \tilde{J}_{t+1}\left(x+f\left(d ; \theta_{k}\right)\right) \\
& =c(d)+\sum_{i=1}^{n} \tilde{J}_{t+1}\left(x+f\left(d ; \theta_{i}\right)\right) p\left(\theta_{i}\right)+\delta \sum_{i=1}^{k-1} p\left(\theta_{i}\right)\left[\tilde{J}_{t+1}\left(x+f\left(d ; \theta_{i}\right)\right)-\tilde{J}_{t+1}\left(x+f\left(d ; \theta_{k}\right)\right)\right] \\
& +\delta \sum_{i=k+1}^{n} p\left(\theta_{i}\right)\left[\tilde{J}_{t+1}\left(x+f\left(d ; \theta_{k}\right)\right)-\tilde{J}_{t+1}\left(x+f\left(d ; \theta_{i}\right)\right)\right] .
\end{aligned}
$$

Now, to prove that the optimal dose is increasing in $\delta$, we show that the function $W_{t}(\cdot, \cdot)$ has decreasing differences. Let $0 \leq \delta^{-} \leq \delta^{+} \leq 1$ and $d^{+}, d^{-} \in D, d^{+} \geq d^{-}$. Let $S=$ $\left[W_{t}\left(d^{+} ; \delta^{+}\right)-W_{t}\left(d^{-} ; \delta^{+}\right)\right]-\left[W_{t}\left(d^{+} ; \delta^{-}\right)-W_{t}\left(d^{-} ; \delta^{-}\right)\right]$. For convenience, denote $\overline{\tilde{J}_{t+1}}\left(x+f\left(d^{+}, \theta_{i}\right)\right)$ by $\tilde{J}^{+i}, \tilde{J}_{t+1}\left(x+f\left(d^{-}, \theta_{i}\right)\right)$ by $\tilde{J}^{-i}$, and so on. Then, noting that terms that are functions of $d$ alone (and not of $\delta$ ) cancel out, $S$ can be written after some algebraic simplification, as

$S=\left(\delta^{+}-\delta^{-}\right) \sum_{i=1}^{k-1} p\left(\theta_{i}\right)\left[\tilde{J}^{+i}-\tilde{J}^{+k}-\tilde{J}^{-i}+\tilde{J}^{-k}\right]+\left(\delta^{+}-\delta^{-}\right) \sum_{i=k+1}^{n} p\left(\theta_{i}\right)\left[\tilde{J}^{+k}-\tilde{J}^{+i}-\tilde{J}^{-k}+\tilde{J}^{-i}\right]$.

We show that the term inside the summation is always non-positive. Consider any two doses $d^{+} \geq d^{-}$and dose-response parameters $\theta^{+} \geq \theta^{-}$. Since the dose-response function is decreasing in dose and the uncertainty parameter $\theta$, one of the following two orderings must hold:

$$
\text { either } f^{++} \leq f^{+-} \leq f^{-+} \leq f^{--}, \quad \text { or } \quad f^{++} \leq f^{-+} \leq f^{+-} \leq f^{--} .
$$

Here, once again, $f^{++}$denotes $f\left(d^{+}, \theta^{+}\right)$, and so on. Further, since $f$ has increasing differences in $(d, \theta)$, it satisfies $f^{--}-f^{-+} \geq f^{+-}-f^{++}$. Then, as $\tilde{J}_{t+1}$ is a convex increasing function, we have, $\tilde{J}^{+-}-\tilde{J}^{++}-\tilde{J}^{--}+\tilde{J}^{-+} \leq 0$. Here, $\tilde{J}^{++} \triangleq \tilde{J}_{t+1}\left(x+f\left(d^{+}, \theta^{+}\right)\right)$, and so on. Thus, the term inside the summation is always non-positive and $W_{t}(\cdot, \cdot)$ has decreasing differences over $D \times[0,1]$.

Decreasing differences imply Theorem 4.2 via a standard argument by contradiction (omitted).

Theorem 4.2 also holds if the dose-response function is monotonically increasing in $\theta$, and has decreasing differences over $X \times D$. This can be shown via a straightforward algebraic modification (omitted) of the above proof. Assumption 4.1 requires that the marginal improvement offered by a larger dose be increasing in the dose-response parameter and hence could be restrictive. Nevertheless, it is a mathematical necessity as we shall see in the next section.

\section{Numerical results}

We conducted experiments for a hypothetical disease with inverse-power response, where state $y_{t}$ evolves as $y_{t+1}=\frac{1}{\left(\theta+d_{t}\right)^{k}} y_{t}$ for some $k>0$. Taking logarithms, and setting $x_{t}=\log y_{t}$, this reduces to $x_{t+1}=x_{t}-k \log \left(\theta+d_{t}\right)$. The function $f(d, \theta)=-k \log (d+\theta)$ satisfies Assumptions 2.1, 3.1, and 4.1. We set $c(d)=c d$; here, as in Kotas and Ghate, $c>0$ is called the coefficient of dose aversion. The terminal disutility function $h(x)$ was chosen to equal $\exp (x)$. Since $x$ is the logarithm of the disease condition $y$, this exponential function amounts to trading-off the total dose given against the final disease state reached as is common in the clinical literature on RGD. These disutility functions were also employed in Kotas and Ghate and they satisfy Assumption 2.2. We report numerical results for $k=1$, using $T=10$ sessions with initial disease condition $x_{1}=1$ and the 
dose levels normalized to $D=[0,1]$. The uncertain dose-response parameters $\Theta_{t}$ are supported on a discretized subset with 61 equally-spaced grid points of the interval $[1,2]$. The nominal pmf is chosen to be a discretized Normal distribution centered at 1.5 on this grid. As in Kotas and Ghate, the value of $c$ is found by solving an inverse optimization problem, so that a dose of 0.5 is optimal for a 10-session deterministic DP with dose-response parameter 1.5. Here, 0.5 and 1.5 are midpoints of the respective intervals. The parameter $\delta$ is varied between 0 and 1 in increments of 0.2 , where $\delta=0$ recovers the nominal DP. The results are in Figures 2(a)-(b).

Figure 2 shows, as expected from Theorems 3.2 and 4.2, that robust optimal doses are increasing in worsening disease conditions and in the level of uncertainty $\delta$. We also found, as expected, that the cost-to-go was increasing in $\delta$ (figure not shown for brevity) because the inner maximization is performed over a larger uncertainty set. This is consistent with Figure 2(b), which shows that the price of robustness is increasing in $\delta$.

Finally, we were able to construct a counterexample (omitted for brevity) using the MichaelisMenten function where the conclusion of Theorem 4.2 was numerically observed to fail because this function was increasing in the dose-response parameter but had increasing differences.

Although our analyses focused on interval uncertainty sets, the robust Bellman's equations (2) allow for other uncertainty sets such as entropy, maximum likelihood, and ellipsoidal. The inner problems for these sets are not LPs but they are convex and, at least for entropy and maximum likelihood, can be solved efficiently via a bisection algorithm [5, 10]. Structural results analogous to Theorem 3.2 and Theorem 4.2, however, might not hold in these cases, as the worst-case pmfs from the inner problems will likely depend in a complicated manner on the state-action pairs.

Kotas and Ghate considered a fixed number $T$ of treatment sessions, as in current guidelines for many diseases including hepatitis $\mathrm{C}$ and cancer. One extension of their nominal DP would be to optimal stopping problems, where the number of treatment sessions is instead determined adaptively based on the uncertain disease evolution. When such a model and its analysis are available in the future, it would be interesting to investigate their robust counterpart.

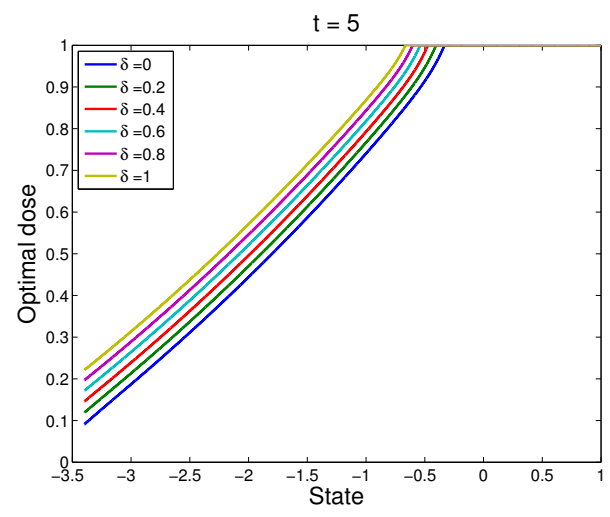

(a)

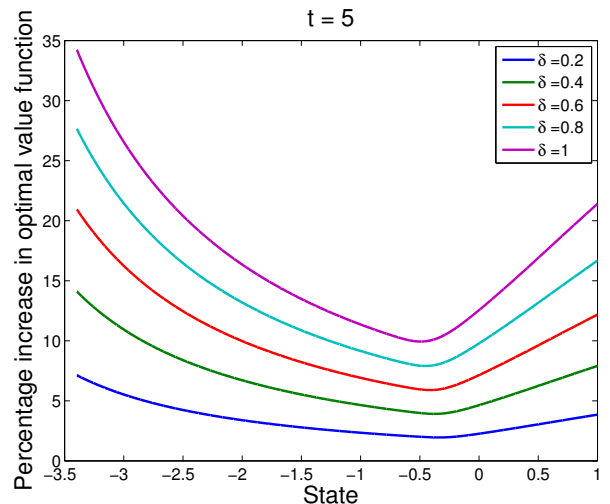

(b)

Figure 2: (a) Robust optimal dose in session $t=5$ (other sessions behaved similarly). As in Theorems 3.2 and 4.2, robust optimal doses are increasing in worsening disease conditions and in increasing dose-response ambiguity. (b) Percentage increase in the robust optimal cost-to- go function over the nominal optimal cost-to-go function for $t=5$ (other sessions behaved similarly). A higher ambiguity in dose-response results in a larger price of robustness. 


\section{Acknowledgment}

Funded in part by the National Science Foundation through \#CMMI 1054026 and \#CMMI 1536717.

\section{References}

[1] A Ben-Tal, L El Ghaoui, and A Nemirovski. Robust Optimization. Princeton University Press, Princeton, NJ, USA, 2009.

[2] S Boyd and L Vandenberghe. Convex Optimization. Cambridge University Press, Cambridge, UK, 2004.

[3] M E Burton, L M Shaw, J J Schentag, and W E Evans. Applied Pharmacokinetics E Pharmacodynamics : Principles of Therapeutic Drug Monitoring. Lippincott Williams \& Wilkins, Baltimore, Maryland, USA, 4th edition, 2006.

[4] Food and Drug Administration. Industry guideline: Dose-response information to support drug registration. Federal Register, 59(216):55972-55976, November 1994.

[5] G N Iyengar. Robust dynamic programming. Mathematics of Operations Research, 30(2):257$280,2005$.

[6] J Kotas and A Ghate. Response-guided dosing for rheumatoid arthritis. IIE Transactions in Healthcare Systems Engineering, 6(1):1-21, 2016.

[7] L B Marks, E D Yorke, A Jackson, R K Ten Haken, L S Constine, A Eisbruch, S M Bentzen, J Nam, and J O Deasy. Use of normal tissue complication probability models in the clinic. International Journal of Radiation Oncology Biology Physics, 76(3):S10-S19, 2010.

[8] S A Murphy, L M Collins, and A J Rush. Customizing treatment to the patient: adaptive treatment strategies. Drug and Alcohol Dependence, 88(Supplement 2):S1-S3, 2007.

[9] National Institutes of Health Office of the Director. NIH consensus statement on management of hepatitis C: 2002. NIH Consens State Sci Statements, 19(3):1-46, 2002.

[10] A Nilim and L El Ghaoui. Robust control of Markov decision processes with uncertain transition matrices. Operations Research, 53(5):780-798, 2005.

[11] A Schumitzky. Application of stochastic control theory to optimal design of dosage regimens. In D Z D'Argenio, editor, Advanced methods of pharmacokinetic and pharmacodynamic systems analysis, volume 1, chapter 13, pages 135-152. Plenum Press, 1991. 